\title{
Robot-assisted vs. Laparoscopic Partial Nephrectomy: utilization rates and perioperative outcomes
}

Jesse D. Sammon, Pierre I. Karakiewicz, Maxine Sun, Praful Ravi, Khurshid R. Ghani, Wooju Jeong, Marco Bianchi, Jens Hansen, Paul Perrotte, James 0. Peabody, Craig G. Rogers, Shahrokh F. Shariat, Mani Menon, Quoc-Dien Trinh

Vattikuti Urology Institute, Henry Ford Health System (JS, KRG, WJ, JOP, CGR, MM, QDT), Detroit, MI, USA; Cancer Prognostics and Health Outcomes Unit, University of Montreal Health Center (PIK, MS, MB, JH, PP, QDT), Montreal, Canada; Department of Urology, University of Cambridge, Cambridge (PR), United Kingdom; Department of Urology, Universita Vita Salute San Raffaele (MB), Milan, Italy; Martini-Clinic, Prostate Cancer Center Hamburg-Eppendorf (JH, SFS), Hamburg, Germany

\section{ABSTRACT}

Objectives: To examine the effect of surgical approach on perioperative morbidity and mortality after partial nephrectomy.

Materials and Methods: Within the Nationwide Inpatient Sample, patients who underwent RAPN or LPN between October 2008 and December 2009 were identified. Propensity-based matching was performed to adjust for potential baseline differences between the two groups. The rates of intraoperative and postoperative complications, blood transfusions, prolonged length of stay, and in-hospital mortality, stratified according to RAPN vs. LPN, were compared.

Results: Overall, 851 (72.5\%) patients underwent RAPN and 323 (27.5\%) underwent LPN. For RAPN and LPN respectively, the following rates were recorded in the propensity-score matched cohort: blood transfusions, 4.5 vs. $6.8 \%(\mathrm{p}=0.223)$; intraoperative complications, 5.2 vs. $2.6 \%(p=0.096)$; postoperative complications, 10.6 vs. $13.5 \%$ ( $p=0.268$; prolonged length of stay, 6.8 vs. $9.4 \%$ ( $p=0.238$ ); in-hospital mortality, 0.0 vs. $0.0 \%$. Conclusions: RAPN has supplanted LPN as the predominant minimally invasive surgical approach for renal masses. Perioperative outcomes after RAPN and LPN are comparable. Interpretation of these findings needs to take into account the lack of adjustment for case complexity and surgical expertise.

\section{ARTICLE INFO}

\section{Key words:}

Nephrectomy; Robotics;

Laparoscopy

Int Braz J Urol. 2013; 39: 377-86

Submitted for publication:

March 27, 2012

Accepted after revision:

April 24, 2013

\section{INTRODUCTION}

The incidence of renal cell carcinoma (RCC) has steadily risen in the United States in recent years. While the rates of metastatic and regional disease have remained steady, the incidence of organ confined disease has risen (1). Increased utilization of cross-sectional imaging has led to more frequent discovery of suspicious renal masses for which surgical resection remains the standard of care (2).

Over the past decade, the management of suspicious renal masses in patients with a healthy contra-lateral renal unit has undergone a fundamental evolution; techniques developed for imperative partial nephrectomy have now become the standard of care. Due to the downward stage migration in contemporary years $(1,3)$, utilization 
rate of partial nephrectomy (PN) has increased significantly (4). PN has been demonstrated to provide equivalent oncologic control (5), improved renal function, a lower risk of cardiovascular disease, as well as improved overall survival (6-8). In consequence, PN is supported by both American and European guidelines when indicated and technically feasible.

Laparoscopic PN (LPN) is a minimally invasive alternative to open partial nephrectomy $(9,10)$. However, LPN is technically challenging, limiting its widespread adoption. Robot-assisted PN (RAPN) has emerged as a viable alternative to mitigate the technical challenges of LPN, demonstrating perioperative outcomes at least comparable to LPN, with the benefit of reduced warm ischemia time (11-13).

In the current manuscript, we explore the effect of minimally invasive surgical approach (RAPN vs. LPN) on five immediate and short-term PN outcomes. Specifically, we focus on blood transfusions, on intraoperative and postoperative complications, on prolonged length of stay (LOS) beyond the 75th percentile, and on in-hospital mortality. We rely on propensity-score matched analyses to adjust for potential differences between patients undergoing RAPN or LPN, using a contemporary (2008-2009) population-based cohort of individuals.

\section{MATERIALS AND METHODS}

\section{Data Source}

Data from the Nationwide Inpatient Sample (NIS) were abstracted between 0ctober 2008 and December 2009. The NIS includes inpatient discharge data collected via federal-state partnerships, as part of the Agency for Healthcare Research and Quality's Healthcare Cost and Utilization Project. As of the year 2009 , the NIS contains data from approximately 8 million hospital stays drawn from more than 1,000 community hospitals in 44 States, approximating $20 \%$ of community hospitals within the United States, including public hospitals and academic medical centers. The NIS is the sole hospital database in the United States with charge information on all patients regardless of payer, including persons covered by Medicare, Medicaid, private insurance, and the uninsured.

\section{Sample population and surgical procedures}

Using a previously described extraction methodology (14), patients with a primary diagnosis of cancer of the kidney were identified using the International Classification of Disease, 9th Revision, Clinical Modification (ICD-9-CM) diagnostic code: 189.0; patients who underwent PN (55.4) were abstracted. Secondary diagnostic codes (ICD-9-CM 197.0, 197.7, 198.x) were used to identify patients with metastases, who were subsequently excluded from analysis. Beginning October 1, 2008, the robot-assisted modifier (ICD-9-CM 17.4x) code was recognized by the National Center of Health Statistics and the Centers for Medicare and Medicaid Services and was used to identify RAPN. Laparoscopic surgical approach was identified via concurrent procedure codes for laparoscopic exploration (54.21). The remaining patients, with an absence of RAPN or LPN codes, were determined as having undergone an OPN, and were excluded from further analysis $(n=2921)$.

\section{Baseline patient and hospital characteristics}

Patient characteristics include age at surgery (in years), coded as a continuous variable. Gender and race (white, black and other races including: Hispanic, Asian, Pacific Islander, Native American, or other/unknown) were also examined. Baseline Charlson Comorbidity Index (CCI) was calculated according to Charlson et al. (15), and adapted according to Deyo et al. (16). To ensure uniformity of coding across data sources, detailed insurance categories are combined in the more general groups, namely: private insurance, Medicare, Medicaid, and other (self-pay).

Hospital characteristics include location (rural vs. urban), region (Northeast, Midwest, South, West), and academic status (academic vs. non-academic), and were obtained from the American Hospital Association Annual Survey of Hospitals. Having an American Medical Association (AMA) -approved residency program, membership in the Council of Teaching Hospitals or having a ratio of full-time equivalent interns and residents to beds of 0.25 or higher defined the hospital academic status. Hospital volume 
was defined according to the number of partial nephrectomies performed annually.

Intraoperative complications, postoperative complications, and blood transfusions during hospitalization

The NIS records up to 15 diagnoses and procedures per in-hospital stay. The presence of any complication was defined using ICD-9 diagnoses 2 through 15 . The specific ICD-9 codes used for complications relied on previously published methodology (17). Intraoperative complication was defined as accidental puncture or laceration during a procedure. Additionally, seven groups of postoperative complications were identified, namely: cardiac, respiratory, neurologic, urinary, vascular, infection, hemorrhage, operative wound, as well as septicemia (18). Blood transfusion recipients were identified using the ICD-9 procedure for transfusion of homologous blood (99.04). For purposes of statistical analysis of overall complication and transfusion rates, we stratified patients by 0 vs. 1 or greater, during hospitalization.

\section{LOS and in-hospital mortality}

LOS, provided by the NIS, is calculated by subtracting the admission date from the discharge date. In-hospital mortality information is coded from disposition of patient. Patients with missing or invalid LOS or in-hospital mortality status were not considered within the current study $(\mathrm{n}=10)$. Prolonged LOS was defined as a hospitalization beyond the 75th percentile cut-off of five days.

\section{Statistical analysis}

Descriptive statistics focused on frequencies and proportions for categorical variables. Means, medians and ranges were reported for continuously coded variables. Chi-square and independent-sample $t$ tests were used to compare the statistical significance of differences in respectively proportions and means.

Due to inherent differences between patients undergoing robotic vs. laparoscopic nephrectomy in terms of baseline patient and hospital characteristics, we relied on propensity-score matched analysis $(19,20)$. Cohorts were matched by patient characteristics: age, gender, race, CCI and insurance status; as well as hospital characteristics: volume, location, region, and academic status. Covariate balance between the matched groups was subsequently examined. Chi-square tests were used to compare the statistical significance of differences between outcomes of RAPN and LPN.

Categorical distributions are reported as counts (\%) and continuous variables as means and standard deviations (SD), and medians and interquartile ranges (IQR). Fisher's exact test, Chi-square test, and Mantel-Haenszel test were used to assess differences in distribution among categorical variables. The median test and t-test were used to assess the difference in distributions among continuous variables. All tests were two-sided with a statistical significance set a $p<0.05$. Analyses were conducted using the $\mathrm{R}$ statistical package (the $\mathrm{R}$ foundation for Statistical Computing, version 2.14.1).

\section{RESULTS}

\section{Description of sample population}

Within the NIS, 1174 patients underwent minimally invasive PN between October 2008 and December 2009. Of those, 851 (72.5\%) and $323(27.5 \%)$ patients underwent RAPN and LPN, respectively. Characteristics of the patient populations are presented in Table-1a. Patient populations differed by race; a greater percentage of LPN patients were white (57.6\% vs. $56.2 \%)$. No difference was noted between groups when stratification was performed according to gender, comorbidity, insurance status or income quartile. Hospital characteristics were significantly different between the two groups. Specifically, a higher proportion of RAPNs was performed at non-teaching institutions ( 26.2 vs. $17.6 \%, p=0.002$ ). RAPNs were more often performed in the Midwest (39.1\%), whereas LPNs were most commonly performed in the Northeast (37.2\%).

Propensity-score matching resulted in a cohort of 310 RAPN and 310 LPN patients (Table$-1 b)$. The standardized differences between the two groups in patient and hospital characteristics were less than 10\% (19), indicating a high degree of similarity in the distribution of both 
Table 1a - Demographic characteristics of patients undergoing minimally invasive partial nephrectomy - entire cohort [p values all Chi-Square].

\begin{tabular}{|c|c|c|c|c|}
\hline & Overall & Laparoscopic & Robotic & $\mathrm{p}$ \\
\hline Number of patients & 1174 & 323 & 851 & \\
\hline Year of surgery & & & & 0.760 \\
\hline 2008 & $386(32.9)$ & $104(32.2)$ & $282(33.1)$ & \\
\hline 2009 & $788(67.1)$ & $219(67.8)$ & $569(66.9)$ & \\
\hline Gender & & & & 0.121 \\
\hline Male & $653(55.9)$ & $167(52.2)$ & $486(57.2)$ & \\
\hline Female & $516(44.1)$ & $153(47.8)$ & $363(42.8)$ & \\
\hline Age & & & & 0.545 \\
\hline Mean (SD) & $56.9(14.0)$ & $56.5(14.9)$ & $57.1(13.7)$ & \\
\hline Median (IQR) & $58.0(48.67)$ & $57.0(47.67)$ & $58.0(48.67)$ & \\
\hline Race & & & & 0.012 \\
\hline White & $664(56.6)$ & $186(57.6)$ & $478(56.2)$ & \\
\hline Black & $55(4.7)$ & $25(7.7)$ & $30(3.5)$ & \\
\hline Other & $128(10.9)$ & $33(10.2)$ & $95(11.2)$ & \\
\hline Unknown & 327 (27.9) & $79(24.5)$ & $248(29.1)$ & \\
\hline CCI & & & & 0.762 \\
\hline 0 & $762(64.9)$ & $217(67.2)$ & $545(64.0)$ & \\
\hline 1 & $295(25.1)$ & $77(23.8)$ & $218(25.6)$ & \\
\hline 2 & $70(6.0)$ & $18(5.6)$ & $52(6.1)$ & \\
\hline$\geq 3$ & $47(4.0)$ & $11(3.4)$ & $36(4.2)$ & \\
\hline Income & & & & 0.058 \\
\hline 1st quartile & $210(18.4)$ & $63(20.0)$ & $147(17.8)$ & \\
\hline 2nd quartile & $320(28.0)$ & $80(25.4)$ & $240(29.0)$ & \\
\hline 3rd quartile & $318(27.8)$ & $76(24.1)$ & $242(29.2)$ & \\
\hline 4th quartile & $295(25.8)$ & $96(30.5)$ & $199(24.0)$ & \\
\hline Hospital location & & & & 0.236 \\
\hline Rural & $27(2.3)$ & $10(3.2)$ & $17(2.0)$ & \\
\hline Urban & $1131(97.7)$ & $303(96.8)$ & $828(98.0)$ & \\
\hline Hospital region & & & & $<0.001$ \\
\hline Northeast & $258(22.0)$ & $120(37.2)$ & $138(16.2)$ & \\
\hline Midwest & $429(36.5)$ & $96(29.7)$ & $333(39.1)$ & \\
\hline South & $278(23.7)$ & $66(20.4)$ & $212(24.9)$ & \\
\hline West & $209(17.8)$ & $41(12.7)$ & $168(19.7)$ & \\
\hline Hospital teaching status & & & & 0.002 \\
\hline Non-teaching & $276(23.8)$ & $55(17.6)$ & $221(26.2)$ & \\
\hline Teaching & $882(76.2)$ & $258(82.4)$ & $624(73.8)$ & \\
\hline Hospital volume & & & & 0.085 \\
\hline Mean (SD) & $72(11)$ & $81(121)$ & $69(101)$ & \\
\hline Median (IQR) & 30 (13.59) & 27 (12.48) & $32(14.59)$ & \\
\hline Insurance status & & & & 0.498 \\
\hline Private & $714(60.8)$ & $204(63.2)$ & $510(59.9)$ & \\
\hline Medicaid & $56(4.8)$ & $16(5.0)$ & $40(4.7)$ & \\
\hline Medicare & $350(29.8)$ & $86(26.6)$ & $264(31.0)$ & \\
\hline Other & $54(4.6)$ & $17(5.3)$ & $37(4.3)$ & \\
\hline
\end{tabular}


Table 1b - Demographic characteristics of patients undergoing minimally invasive partial nephrectomy - propensity-score matched cohort [p values all Chi-Square].

\begin{tabular}{|c|c|c|c|c|}
\hline & Overall & Laparoscopic & Robotic & $p$ \\
\hline Number of patieants & 620 & 310 & 310 & \\
\hline Year of surgery & & & & 0.299 \\
\hline 2008 & 194 (31.3) & $103(33.2)$ & $91(29.4)$ & \\
\hline 2009 & $426(68.7)$ & 207 (66.8) & $219(70.6)$ & \\
\hline Gender & & & & 0.519 \\
\hline Male & $334(53.9)$ & $163(52.6)$ & $171(55.2)$ & \\
\hline Female & $286(46.1)$ & $147(47.4)$ & $139(44.8)$ & \\
\hline Age & & & & 0.633 \\
\hline Mean(SD) & $56.3(14.1)$ & $56.5(14.9)$ & $56.0(13.3)$ & \\
\hline Median(IQR) & $57(48.66)$ & $57(47.67)$ & $57(48.65)$ & \\
\hline Race & & & & 0.043 \\
\hline White & $358(57.7)$ & $176(56.8)$ & $182(58.7)$ & \\
\hline Black & $37(6.0)$ & $25(8.1)$ & $12(3.9)$ & \\
\hline Other & $56(9.0)$ & $33(10.6)$ & $23(7.4)$ & \\
\hline Unknown & $169(27.3)$ & $76(24.5)$ & $93(30.0)$ & \\
\hline CCI & & & & 0.983 \\
\hline 0 & $419(67.6)$ & $208(67.1)$ & $211(68.1)$ & \\
\hline 1 & $147(23.7)$ & $75(24.2)$ & $72(23.2)$ & \\
\hline 2 & $35(5.6)$ & $17(5.5)$ & $18(5.8)$ & \\
\hline$\geq 3$ & $19(3.1)$ & $10(3.2)$ & $9(2.9)$ & \\
\hline Income & & & & 0.211 \\
\hline 1st quartile & $117(19.4)$ & 60 (19.9) & $57(18.9)$ & \\
\hline 2nd quartile & $159(26.3)$ & $74(24.5)$ & $85(28.1)$ & \\
\hline 3rd quartile & $159(26.3)$ & $73(24.2)$ & $86(28.5)$ & \\
\hline 4th quartile & $169(28.0)$ & $95(31.5)$ & $74(24.5)$ & \\
\hline Hospital location & & & & 0.664 \\
\hline Rural & $22(3.5)$ & $10(3.2)$ & $12(3.9)$ & \\
\hline Urban & $598(96.5)$ & $300(96.8)$ & $298(96.1)$ & \\
\hline Hospital region & & & & 0.002 \\
\hline Northeast & $223(36.0)$ & $120(38.7)$ & $103(33.2)$ & \\
\hline Midwest & $214(34.5)$ & $86(27.7)$ & $128(41.3)$ & \\
\hline South & $108(17.4)$ & $66(21.3)$ & $42(13.5)$ & \\
\hline West & $75(12.1)$ & $38(12.3)$ & $37(11.9)$ & \\
\hline Hospital teaching status & & & & 0.587 \\
\hline Non-teaching & $101(16.3)$ & $53(17.1)$ & $48(15.5)$ & \\
\hline Teaching & $519(83.7)$ & $257(82.9)$ & $262(84.5)$ & \\
\hline Hospital volume & & & & 0.769 \\
\hline Mean (SD) & $84(120)$ & $83(124)$ & $86(116)$ & \\
\hline Median (IQR) & $31(13.59)$ & $27(11.48)$ & $36(16.60)$ & \\
\hline Insurance status & & & & 0.769 \\
\hline Private & $391(63.1)$ & $197(63.5)$ & $194(62.6)$ & \\
\hline Medicaid & $27(4.4)$ & $15(4.8)$ & $12(3.9)$ & \\
\hline Medicare & $173(27.9)$ & $82(26.5)$ & $91(29.4)$ & \\
\hline Other & $29(4.7)$ & $16(5.2)$ & $13(4.2)$ & \\
\hline
\end{tabular}


populations. The matched populations represent the focus of all subsequent analyses.

Intraoperative and postoperative outcomes during hospitalization

Table-2 shows the rate of intraoperative and postoperative outcomes, stratified according to RAPN and LPN, pre (Table-2a) and post-propensity score matching (Table-2b). In the post propensity-score matched cohort, the rates of intraoperative and postoperative complications were found to be equivalent, as was the rate of blood transfusion and prolonged LOS $(p>0.05)$. Statistically significant differences were however seen for individual complications. Specifically, patients undergoing RAPN had fewer neurologic ( 0.0 vs. $2.3 \%, p=0.008$ ), urinary (0.0 vs. $1.9 \%, p=0.014$ ) and bleeding complications (0.6 vs. $2.9 \%$ ).

\section{DISCUSSION}

RAPN was initially described in 2004 (21) and represents an acceptable alternative to open or laparoscopic PN for selected renal masses. It has been hypothesized that RAPN may help overcome the technical challenges of LPN and may offer an easier transition to minimally invasive PN (22). In this retrospective case-control study, we compare short-term perioperative outcomes of RAPN vs. LPN, relying on a contemporary (20082009) population-based cohort of individuals undergoing minimally invasive partial nephrectomy. To account for baseline patient and hospital differences between the two procedure cohorts, we relied on propensity-score matched analysis to eliminate the plausible biases associated with treatment selection.

Our findings are several-fold. First, our contemporary analysis shows that RAPN has overtaken LPN as the preferred surgical approach of minimally invasive PN in the United States. In this 20\% representative sample of all inpatient admissions between October 2008 and December 2009, nearly three times as many RAPNs were performed compared to LPNs (851 vs. 323), thus accounting for $72.5 \%$ of minimally invasive PNs. This finding is both novel and unexpected; the unique identifier for robotic procedures was only introduced by CMS in 2008; 2009 represents its first full year of utilization. Although objective evidence has shown that the utilization of robot-assisted radical prostatectomy has increased dramatically in recent years $(23,24)$, no previous reported has alluded to a similar phenomenon in the context of PN.

Second, the characteristics of RAPN vs. LPN cases differed regarding several patient and hospital attributes. When compared to LPN patients, patients undergoing RAPN were less likely to be white and less likely to be treated at teaching, high-volume institutions. Moreover, LPN is predominantly performed in the Northeast, while RAPN is chiefly performed in the Midwest. LPN regionalization may be explained by the location of teaching vs. non-teaching hospital within the current database. Specifically, most teaching hospitals were located within the Northeast region, and since the majority of LPN was performed at teaching hospitals, the observed regional variability was likely a reflection of teaching vs. non-teaching hospital status. In consequence, it may be possible that lack of surgical LPN expertise in some areas could have prevented LPN being performed. In this regard, we corroborate previous findings that the use of PN is highly regionalized (25). Taken together, these findings corroborate the hypothesis that the diffusion of laparoscopy has not been uniform and is strongly influenced by patient provider characteristics, such as hospital volume and teaching status (26). Conversely, our findings also indicate that RAPN might have a different dissemination pattern, as this procedure has already been adopted in a higher proportion by surgeons at non-teaching and lower volume institutions than conventional laparoscopy. It is reasonable to presume that introduction of RAPN may in fact accelerate the utilization of PN as patients and surgeons seek to utilize minimally invasive approaches to PN. Unfortunately, temporal trend analyses are not possible since the robot-assisted modifier was only introduced in the fall of 2008.

Nonetheless, these findings emphasize the need for meticulous adjustment of patient differences when comparing perioperative outcomes. Lack of adjustment may result in important confounding of results. Specifically, based on previous 
Table 2a - Outcomes of patients undergoing minimally invasive partial nephrectomy - entire cohort (unmatched).

\begin{tabular}{lcccc}
\hline & Overall & Laparoscopic & Robotic & $p$ \\
\hline Number of patients & 1174 & 323 & 851 & - \\
Intraoperative complication & $43(3.7)$ & $8(2.5)$ & $35(4.1)$ & 0.183 \\
Postoperative complication & & & & \\
Overall & $144(12.3)$ & $42(13.0)$ & $102(12.0)$ & 0.635 \\
Cardiac & $14(1.2)$ & $3(0.9)$ & $11(1.3)$ & 0.608 \\
Respiratory & $46(3.9)$ & $18(5.6)$ & $28(3.3)$ & 0.072 \\
Neurologic & $17(1.4)$ & $7(2.2)$ & $10(1.2)$ & 0.204 \\
Urinary & $14(1.2)$ & $6(1.9)$ & $8(0.9)$ & 0.196 \\
Vascular & $1(0.1)$ & $0(0.0)$ & $1(0.1)$ & 0.538 \\
Infection & $1(0.1)$ & $0(0.0)$ & $1(0.1)$ & 0.538 \\
Hemorrhage & $22(1.9)$ & $9(2.8)$ & $13(1.5)$ & 0.155 \\
Operative wound & $8(0.7)$ & $1(0.3)$ & $7(0.8)$ & 0.340 \\
Septicemia & $1(0.1)$ & $0(0.0)$ & $1(0.1)$ & 0.538 \\
In-hospital mortality & $0(0.0)$ & $0(0.0)$ & $0(0.0)$ & - \\
Blood transfusion & $74(6.3)$ & $22(6.8)$ & $52(6.1)$ & 0.659 \\
Length of stay & & & & $52(6.1)$ \\
\hline Length of stay $>5$ days (median) & $82(7.0)$ & $30(9.3)$ & 0.056 \\
\hline
\end{tabular}

reports, better surgical outcomes are expected in patients treated at teaching (27) and high-volume (28) centers. To minimize bias and control for the baseline differences between RAPN and LPN patients, we relied on propensity-score matching. Propensity-score matching resulted in highly comparable patient and institutional characteristics within the RAPN and LPN groups. In propensity-score matched cohorts, no statistically significant difference between the two groups was recorded with regard to intraoperative and postoperative complications, prolonged LOS and in-hospital mortality. It is noteworthy hemorrhagic complications were significantly lower in the RAPN group ( $p=0.033)$; though no statistically significant difference in transfusion rates was recorded ( $p=0.223$ ).

Based on the diffusion pattern of RAPN, it can be hypothesized that the utilization rates of nephron-sparing surgery will improve. Dulabon et al. (4) used a population-based dataset and identified 18330 localized RCC patients between years 1999 to 2006. PN utilization rates were $21 \%$ in 1999 vs. $45 \%$ in 2006. These findings strongly contrasts with those of Thompson et al. (29), who showed that PN rates at the Memorial Sloan-Kettering Cancer Center increased from 69 to 89\% between 2000 and 2007. The disparities in reported rates highlight the difficult diffusion of LPN for small renal masses, as this technique is relatively challenging and often limited to centers of excellence.

From a practical perspective, our results indicate that on average, similar intraoperative and postoperative outcomes, including transfusion rates, prolonged LOS and in-hospital mortality, are expected whether the patient undergoes RAPN or LPN. However, these results should be interpreted with caution, since the NIS is unable to account for disease characteristics. Specifically, it is not known if complex cases, such as endophytic or hilar tumors, higher stage and grade lesions, are more often performed by the robotic or laparoscopic approach. 
Table $2 \mathrm{~b}$ - Outcomes of propensity-score matched patients undergoing minimally invasive partial nephrectomy. Patients were matched by age, gender, race, CCI and insurance status; as well as by hospital characteristics: volume, location, region, and academic status.

\begin{tabular}{lcccc}
\hline & Overall & Laparoscopic & Robotic & $p$ \\
\hline Number of patients & 620 & 310 & 310 & - \\
Intraoperative complication & $24(3.9)$ & $8(2.6)$ & $15(5.2)$ & 0.096 \\
Postoperative complication & & & & \\
Overall & $75(12.1)$ & $42(13.5)$ & $33(10.6)$ & 0.268 \\
Cardiac & $10(1.6)$ & $3(1.0)$ & $7(2.3)$ & 0.202 \\
Respiratory & $29(4.7)$ & $18(5.8)$ & $11(3.5)$ & 0.183 \\
Neurologic & $7(1.1)$ & $7(2.3)$ & $0(0.0)$ & 0.008 \\
Urinary & $6(1.0)$ & $6(1.9)$ & $0(0.0)$ & 0.014 \\
Vascular & $0(0.0)$ & $0(0.0)$ & $0(0.0)$ & - \\
Infection & $0(0.0)$ & $0(0.0)$ & $0(0.0)$ & - \\
Hemorrhage & $11(1.8)$ & $9(2.9)$ & $2(0.6)$ & 0.033 \\
Operative wound & $1(0.2)$ & $1(0.3)$ & $0(0.0)$ & 0.317 \\
Septicemia & $1(0.2)$ & $0(0.0)$ & $1(0.3)$ & 0.317 \\
In-hospital mortality & $0(0.0)$ & $0(0.0)$ & $0(0.0)$ & - \\
Blood transfusion & $35(5.6)$ & $21(6.8)$ & $14(4.5)$ & 0.223 \\
Length of stay & & & & \\
Length of stay > 5 days (median) & $50(8.1)$ & $29(9.4)$ & $21(6.8)$ & 0.238 \\
\hline
\end{tabular}

To the best of our knowledge, the current manuscript represents the first population-based report to compare complication rates after RAPN and LPN. Benway et al. recently published the largest multi-institutional comparison of the two approaches, describing the outcomes of three experienced minimally invasive surgeons $(\mathrm{n}=247)(11)$. They found similar post-operative complication rates of RAPN vs. LPN (8.6 vs. 10.2\%), which corroborate the findings of this study.

A significant limitation of the NIS is the lack of capture of tumor stage and pathological characteristics; there was likely some variability in distribution and this would affect our findings. There may have also been unobserved differences in operative characteristics that were not captured in discharge level data, ie. warm ischemia time. Although propensity-based matching was performed, there may have also been differences amongst the groups concerning important patient variables (personal preferences, education, body mass index and medication utilization), as well as socio-economical determinants. Moreover, our mortality estimates are based on in-hospital rates. It is possible that the true mortality is underestimated as some patients may have died at other institutions where their mortality was not captured. Finally, we were not able to classify complications according to ideal, prospective methodologies, such as the Clavien classification, nor were we able to grade them using the Common toxicity Criteria for Adverse Events $(30,31)$.

\section{CONCLUSIONS}

RAPN has supplanted LPN as the predominant minimally invasive surgical approach for renal 
masses. Perioperative outcomes after RAPN and LPN are comparable and remain comparable following adjustment for several common confounders. Interpretation of these findings needs to take into account the lack of adjustment for case complexity and surgical expertise.

\section{ACKNOWLEDGMENTS}

Pierre I Karakiewicz is partially supported by the University of Montreal Health Centre Urology Specialists, Fonds de la Recherche en Santé du Québec, the University of Montreal Department of Surgery and the University of Montreal Health Centre (CHUM) Foundation.

\section{CONFLICT OF INTEREST}

None declared.

\section{REFERENCES}

1. Sun M, Thuret R, Abdollah F, Lughezzani G, Schmitges J, Tian $Z$, et al.: Age-adjusted incidence, mortality, and survival rates of stage-specific renal cell carcinoma in North America: a trend analysis. Eur Urol. 2011; 59: 135-41.

2. NCCN: Clinical Practice Guidelines in Oncology: Kidney Cancer. Avaliable at http://www.nccn.org/professionals/physician_gls/f_guidelines.asp Accessed: August 15, 2011.

3. Doeuk N, Guo DY, Haddad R, Lau H, Woo HH, Bariol S, et al.: Renal cell carcinoma: stage, grade and histology migration over the last 15 years in a large Australian surgical series. BJU Int. 2011; 107: 1381-5.

4. Dulabon LM, Lowrance WT, Russo P, Huang WC: Trends in renal tumor surgery delivery within the United States. Cancer. 2010; 116: 2316-21.

5. Van Poppel H, Da Pozzo L, Albrecht W, Matveev V, Bono A, Borkowski $A$, et al.: A prospective, randomised EORTC intergroup phase 3 study comparing the oncologic outcome of elective nephron-sparing surgery and radical nephrectomy for low-stage renal cell carcinoma. Eur Urol. 2011; 59: 543-52.

6. Huang WC, Elkin EB, Levey AS, Jang TL, Russo P: Partial nephrectomy versus radical nephrectomy in patients with small renal tumors--is there a difference in mortality and cardiovascular outcomes? J Urol. 2009; 181: 55-61; discussion 61-2.

7. Thompson RH, Boorjian SA, Lohse CM, Leibovich BC, Kwon ED, Cheville JC, et al.: Radical nephrectomy for pT1a renal masses may be associated with decreased overall survival compared with partial nephrectomy. J Urol. 2008; 179: 46871; discussion 472-3.
8. Weight CJ, Larson BT, Fergany AF, Gao T, Lane BR, Campbell SC, et al.: Nephrectomy induced chronic renal insufficiency is associated with increased risk of cardiovascular death and death from any cause in patients with localized cT1b renal masses. J Urol. 2010; 183: 1317-23.

9. McDougall EM, Clayman RV, Chandhoke PS, Kerbl K, Stone AM, Wick MR, et al.: Laparoscopic partial nephrectomy in the pig model. J Urol. 1993; 149: 1633-6.

10. Winfield HN, Donovan JF, Godet AS, Clayman RV: Laparoscopic partial nephrectomy: initial case report for benign disease. J Endourol. 1993; 7 : 521-6.

11. Benway BM, Bhayani SB, Rogers CG, Dulabon LM, Patel MN, Lipkin $M$, et al.: Robot assisted partial nephrectomy versus laparoscopic partial nephrectomy for renal tumors: a multiinstitutional analysis of perioperative outcomes. J Urol. 2009; 182: 866-72.

12. Wang AJ, Bhayani SB: Robotic partial nephrectomy versus laparoscopic partial nephrectomy for renal cell carcinoma: single-surgeon analysis of $>100$ consecutive procedures. Urology. 2009; 73: 306-10.

13. Rogers CG, Metwalli A, Blatt AM, Bratslavsky G, Menon M, Linehan WM, et al.: Robotic partial nephrectomy for renal hilar tumors: a multi-institutional analysis. J Urol. 2008; 180: 23536; discussion 2356 .

14. Murphy MM, Witkowski ER, Ng SC, McDade TP, Hill JS, Larkin AC, et al.: Trends in adrenalectomy: a recent national review. Surg Endosc. 2010; 24: 2518-26.

15. Charlson ME, Pompei P, Ales KL, MacKenzie CR: A new method of classifying prognostic comorbidity in longitudinal studies: development and validation. J Chronic Dis. 1987; 40: 373-83.

16. Deyo RA, Cherkin DC, Ciol MA: Adapting a clinical comorbidity index for use with ICD-9-CM administrative databases. J Clin Epidemiol. 1992; 45: 613-9.

17. Hu JC, Gu X, Lipsitz SR, Barry MJ, D’Amico AV, Weinberg AC, et al.: Comparative effectiveness of minimally invasive vs open radical prostatectomy. JAMA. 2009; 302: 1557-64.

18. Allareddy V, Allareddy V, Konety BR: Specificity of procedure volume and in-hospital mortality association. Ann Surg. 2007; 246: 135-9.

19. D'Agostino RB Jr: Propensity score methods for bias reduction in the comparison of a treatment to a non-randomized control group. Stat Med. 1998; 17: 2265-81.

20. Stukel TA, Fisher ES, Wennberg DE, Alter DA, Gottlieb DJ, Vermeulen MJ: Analysis of observational studies in the presence of treatment selection bias: effects of invasive cardiac management on AMI survival using propensity score and instrumental variable methods. JAMA. 2007; 297: 278-85.

21. Gettman MT, Blute ML, Chow GK, Neururer R, Bartsch G, Peschel R: Robotic-assisted laparoscopic partial nephrectomy: technique and initial clinical experience with DaVinci robotic system. Urology. 2004; 64: 914-8. 
22. Sukumar S, Rogers CG: Robot-assisted partial nephrectomy. J Endourol. 2011; 25: 151-7.

23. Hu JC, Gu X, Lipsitz SR, Barry MJ, D’Amico AV, Weinberg AC, et al.: Comparative effectiveness of minimally invasive vs open radical prostatectomy. JAMA. 2009; 302: 1557-64.

24. Trinh QD, Sammon J, Sun M, Ravi P, Ghani KR, Bianchi M, et al.: Perioperative outcomes of robot-assisted radical prostatectomy compared with open radical prostatectomy: results from the nationwide inpatient sample. Eur Urol. 2012; 61: 679-85.

25. Baillargeon-Gagné S, Jeldres C, Lughezzani G, Sun M, Isbarn $\mathrm{H}$, Capitanio U, et al:: A comparative population-based analysis of the rate of partial vs radical nephrectomy for clinically localized renal cell carcinoma. BJU Int. 2010; 105: 359-64.

26. Miller DC, Taub DA, Dunn RL, Wei JT, Hollenbeck BK: Laparoscopy for renal cell carcinoma: diffusion versus regionalization? J Urol. 2006; 176: 1102-6; discussion 1106-7.
27. Trinh QD, Schmitges J, Sun M, Shariat SF, Sukumar S, Bianchi $\mathrm{M}$, et al.: Radical prostatectomy at academic versus nonacademic institutions: a population based analysis. J Urol. 2011; 186: 1849-54.

28. Begg CB, Cramer LD, Hoskins WJ, Brennan MF: Impact of hospital volume on operative mortality for major cancer surgery. JAMA. 1998; 280: 1747-51.

29. Thompson RH, Kaag M, Vickers A, Kundu S, Bernstein M, Lowrance W, et al.: Contemporary use of partial nephrectomy at a tertiary care center in the United States. J Urol. 2009; 181: 993-7.

30. Simmons MN, Gill IS: Decreased complications of contemporary laparoscopic partial nephrectomy: use of a standardized reporting system. J Urol. 2007; 177: 2067-73; discussion 2073.

31. Nogueira L, Katz D, Pinochet R, Godoy G, Kurta J, Savage CJ, et al.: Critical evaluation of perioperative complications in laparoscopic partial nephrectomy. Urology. 2010; 75: 288-94.

\section{Correspondence address:} Dr. Jesse D. Sammon Vattikuti Urology Institute Henry Ford Health System 2799 W. Grand Boulevard Detroit, Michigan, USA, 48202

Fax: + 1313 916-4352 E-mail address: jsammon79@gmail.com 